\title{
Developing multifunctional nanomaterials for alternate/renewable energy applications
}

\author{
Shubra Singh*, Sumithra Sivadas Menon, Suchita and R. Anitha Muthukumar \\ Crystal Growth Centre, Anna University, Chennai -600025 \\ *Email: shubra6@gmail.com
}

Multifunctional oxides have emerged as the most useful compounds in recent times with applications as widespread as sensing, photocatalytic as well as antiviral behavior, all of which can be enhanced by forming nano- and microstructures to increase the ratio of surface area to volume. In this work we discuss the synthesis and application of nanostructured oxides with applications such as photocatalysis, $\mathrm{CO}_{2}$ capture etc. Applications such as Photocatalysis by nanoparticles have a brighter future ahead as they provide tunable high absorption, stability and surfaces area besides being inexpensive and nontoxic. In photocatalytic reactions semiconductors are generally used as catalysts in presence of ultraviolet light and sunlight. Presently not many materials exist with an appropriate band gap position necessary for practical applications. Most photocatalytic materials possess transition-metal cations with a d0 electronic configuration or metal cations with d10 electronic configuration. These ions have empty d or sp orbitals which form the bottom of the conduction bands [1-2]. The top of the valence bands of metaloxide photocatalysts with $\mathrm{d} 0$ - or d10-metal cations consist of $\mathrm{O} 2 \mathrm{p}$ orbitals, which are located at about $+3 \mathrm{eV}$ or higher making the band gap too wide to absorb any visible light.

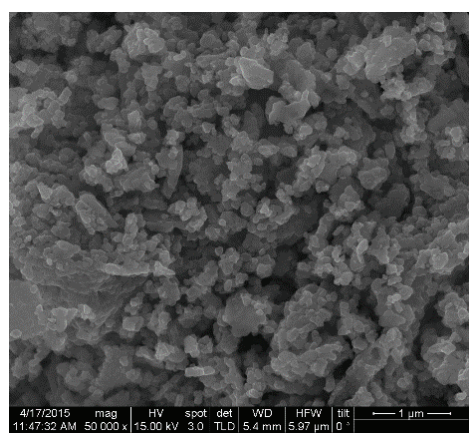

Figure 1: Morphology and microstructure of nanostructured solid solution

In this report we focus our attention on materials which exhibit absorption even in visible light improving their reactivity when synthesized in nanostructured form. Besides this we will also be presenting the role played by another oxide, namely $\mathrm{Ca}_{2} \mathrm{Fe}_{2} \mathrm{O}_{5}$ as nanostructured cathode material for Intermediate temperature SOFCs. Figure 1 and Figure 2 exhibit the Morphology and microstructure of the $\mathrm{Ca}_{2} \mathrm{Fe}_{2} \mathrm{O}_{5}$ nanostructures.
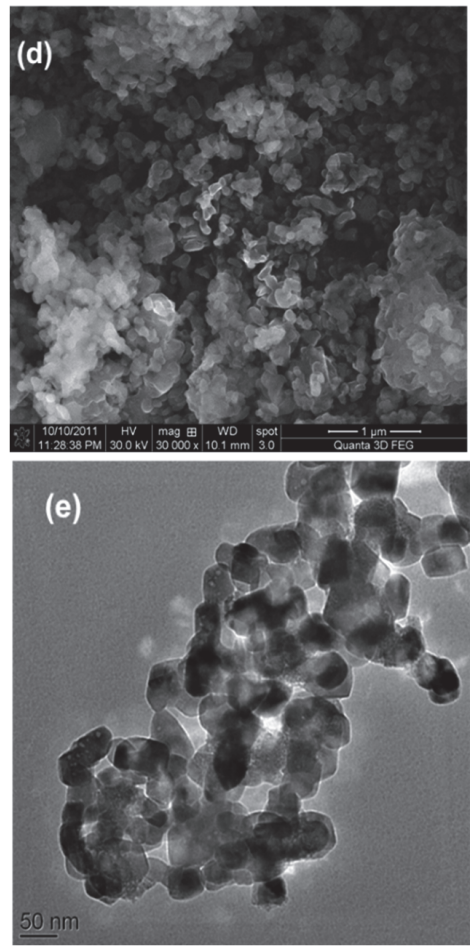

Figure 2: Morphology and microstructure of nanostructured $\mathrm{Ca}_{2} \mathrm{Fe}_{2} \mathrm{O}_{5}$

Acknowledgements: S. Singh would like to acknowledge UGC-FRP Scheme and DST Inspire.

\section{References}

1. Maeda et al., J.Phys.Chem.C, 111(22) (2007) 7851-7861

2. Maeda, Pure Appl. Chem., 78, pp. 2267-2276 (2006). 